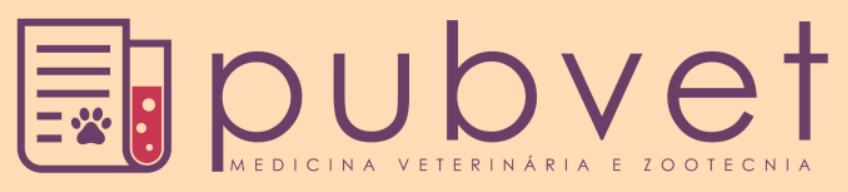

https://doi.org/10.31533/pubvet.v12n10a185.1-16

\title{
Biotécnicas da reprodução aplicada aos bubalinos (Bubalus Bubalis)
}

\author{
Raquel Rodrigues Costa Mello ${ }^{1 *}$, Elizângela Mírian Moreira ${ }^{2}{ }^{\bullet}$, Joaquim Esquerdo \\ Ferreira $^{1} \bullet$, Rosana Colatino Soares Reis ${ }^{3}{ }^{\bullet}$, Marco Roberto Bourg de Mello ${ }^{3} \bullet$
}

${ }^{I}$ Doutorando(a), Departamento de Reprodução e Avaliação Animal, Instituto de Zootecnia, Universidade Federal Rural do Rio de Janeiro, Seropédica, Rio de Janeiro, Brasil ${ }^{2}$ Pós-Doutorando, Bolsista de Desenvolvimento Científico Regional do CNPq - Nível C, Fundação de Amparo ao Desenvolvimento das Ações Científicas e Tecnológicas e à Pesquisa, Empresa Brasileira de Pesquisa Agropecuária, Porto Velho, Rondônia, Brasil

${ }^{3}$ Professor(a) Adjunto(a), Departamento de Produção Animal, Instituto de Zootecnia, Universidade Federal Rural do Rio de Janeiro, Seropédica, Rio de Janeiro, Brasil *Autor para correspondência, E-mail: raquellidda@yahoo.com.br

\begin{abstract}
RESUMO. A criação de búfalos vem se difundindo mundialmente devido à superioridade que pode apresentar em relação aos outros ruminantes domésticos, principalmente a adaptação às diversas condições climáticas do território brasileiro, sendo que ele poderá produzir carne e leite para suprir as necessidades do mercado nacional, propiciando grande contribuição ao atendimento da demanda familiar. A exploração zootécnica dos bubalinos caracteriza-se por apresentar boa eficiência reprodutiva e rápido desenvolvimento ponderal, sendo que este potencial genético pode ser multiplicado mais rapidamente por biotécnicas da reprodução, pois os mercados nacional e internacional de bubalinos necessitam de animais geneticamente superiores. Portanto, devido à importância da criação de bubalinos para o País, o objetivo desta revisão bibliográfica é abordar alguns aspectos da fisiologia reprodutiva e a utilização de biotécnicas reprodutivas nesta espécie, a fim de se promover melhorias para a seleção zootécnica e o desempenho destes animais.
\end{abstract}

Palavras chave: inseminação artificial, melhoramento genético, produtividade, transferência de embriões

\section{Reproductive Biotechniques applied to buffalos (Bubalus Bubalis)}

ABSTRACT. The buffalo breeding is spreading worldwide due to the superiority which may have in relation to other domestic ruminants, especially the adaptation of different climatic conditions of the Brazilian territory, and to produce meat and milk to supply the needs of the national business market, providing great contribution to the supply of family demands. The livestock use of buffaloes is characterized by the good reproductive efficiency and fast body weight gain, and this genetic potential can be multiplied faster by reproductive biotechniques, Buffalo's national and international business markets requires superior genetically animals. Therefore, due to the importance of buffalo breeding for the country, the aim of this review is to describe some aspects of reproductive physiology and the use of reproductive biotechniques in this species, to promote improvements to the genetic selection and performance of these animals.

Key words: artificial insemination, breeding, productivity, embryo transfer

\section{Biotécnica de la reproducción aplicada a los bufalinos (Bubalus Bubalis)}

RESUMEN. La creación de búfalos se viene difundiendo mundialmente debido a la superioridad que puede presentar con relación a los otros rumiantes domésticos, principalmente la adaptación a diversas condiciones climáticas del territorio brasileño, siendo que podrá producir carne y leche para suplir las necesidades del mercado nacional, propiciando gran contribución a la atención de la demanda familiar. La exploración 
zootécnica de los bubalinos se caracteriza por presentar una buena eficiencia reproductiva y un rápido desarrollo de peso, y este potencial genético puede multiplicarse más rápidamente por biotécnicas de reproducción, ya que los mercados nacionales e internacionales de bufalinos necesitan animales genéticamente superiores. Por lo tanto, debido a la importancia de la creación de bufalinos para el país, el objetivo de esta revisión bibliográfica es abordar algunos aspectos de la fisiología reproductiva y la utilización de biotécnicas reproductivas en esta especie, a fin de promover mejoras para la selección zootécnica y el desempeño de estos animales.

Palabras clave: inseminación artificial, mejoramiento genético, productividad, transferencia de embriones

\section{Introdução}

O rebanho brasileiro de bubalinos está estimado em 1,3 milhões de animais de acordo com dados do Ministério da Agricultura, Pecuária e Abastecimento - MAPA (BRASIL, 2017). É o maior do Ocidente e vem apresentando desde 2011 um crescimento significativamente superior ao observado em bovinos, suínos, ovinos e frangos.

As duas principais raças de búfalos no Brasil são o Murrah e o Mediterrâneo que se distribuíam pelas cincos regiões do Brasil nas seguintes proporções: Norte (63\%), Nordeste $(9,5 \%)$, Sudeste $(9,2 \%)$, Sul $(13,5 \%)$ e Centro Oeste $(5,6 \%)$ (IBGE, 2016). Na maioria das regiões a criação é para a produção de carne. São abatidos anualmente 117 mil búfalos, movimentando um mercado de $\mathrm{R} \$ 448$ milhões.

A produção de leite representa outra importante atribuição do búfalo, não somente para o consumo in natura, mas também para o preparo de queijos especiais e manteiga (Tonhati et al., 2000, Rodrigues et al., 2008).

Santana Júnior et al., (2011), Malhado et al., (2012), relataram problemas na estrutura populacional de búfalos no Brasil, aconselhando criadores a introdução de novos indivíduos no rebanho, projetando sistemas de acasalamento que reduzem a consanguinidade. Diante desse contexto, o potencial genético pode ser multiplicado mais rapidamente por biotécnicas da reprodução, como a inseminação artificial (IA), inseminação artificial em tempo fixo (IATF), transferência de embriões (TE), aspiração folicular guiada por ultrassom aliada à produção in vitro de embriões (OPU-PIV) e criopreservação de sêmen e embriões (Baruselli et al., 2007; $\underline{\text { Mello }}$ et al., 2013)

Este trabalho de revisão bibliográfica irá abordar alguns aspectos da fisiologia reprodutiva e a utilização de biotécnicas da reprodução utilizada em bubalinos, a fim de se promover melhorias para a seleção genética e o desempenho produtivo destes animais.

\section{Fisiologia do ciclo estral em bubalinos}

Nas regiões mais distantes da linha do Equador, no sentido norte ou sul, a búfala se comporta como um animal poliestral sazonal de dias curtos, apresentando interrupção da ciclicidade durante o verão, quando a luminosidade diária é maior. Portanto, em regiões próximas a linha do Equador, as fêmeas bubalinas são animais de comportamento poliestral contínuo, ou seja, ciclam durante todo o ano (Vale \& Ribeiro, 2005).

A duração do ciclo estral em bubalinos pode variar de 16 a 33 dias, com duração média de 21 a 24 dias, havendo variação entre as raças (Vale \& Ribeiro, 2005; Siqueira et al., 2009). De fato, Dobson \& Kamonpatana (1986) citam duração média de 21 dias, tal como ocorre nos bovinos. No entanto, alguns autores observaram duração média de 42 dias, quando se somaram os ciclos de duração normal com os de duração anormal (Vale \& Ribeiro, 2005). Essa amplitude se deve a fatores como clima, manejo nutricional e genética. Animais submetidos a manejo nutricional inadequado com deficiência em energia, proteína e minerais têm tendência a apresentar estros curtos ou longos, da mesma maneira quando submetidos ao estresse térmico (Vale \& Ribeiro, 2005).

As fases do ciclo estral nos bubalinos são: proestro, estro, metaestro e diestro, onde o estro varia consideravelmente entre 12 a 36 horas (Jainudeen, 1986; Vale \& Ribeiro, 2005; Siqueira et al., 2009).

Os sintomas de estro nas fêmeas bubalinas não se manifestam como nos bovinos, onde também são variados em termos percentuais de ocorrência, desde a presença de edema da vulva, descarga de muco, micção frequente, cervix aberta e aumento 
de tônus uterino. A melhor forma de detecção do estro na búfala é a utilização de rufiões com buçal marcador, associada à frequente observação visual (Singh et al., 1984; Vale, 1988; Siqueira et al., 2009). Um dos sinais de estros mais seguros observado por esses autores foi a aceitação de monta pelo rufião, evidente em $100 \%$ dos casos. Com a aproximação do estro, as fêmeas tornam-se impacientes, andam ao redor dos piquetes, levantam a cabeça, movimentam a cauda, apresentam comportamento homossexual (montam ou deixam ser montadas por outras), mugido e micção frequente (Vale \& Ribeiro, 2005). A maior concentração dos estros é noturna, com $84 \%$ dos animais aceitando a monta das 18 às 6 horas (Siqueira et al., 2009). Observa-se que a ocorrência de estro silencioso e anovulatório é frequente na búfala, principalmente em animais submetidos à manejo nutricional deficiente ou expostos a estresse térmico (Vale et al., 1991).

Segundo Vale \& Ribeiro (2005), a atividade homossexual nessa espécie não é tão comum, registrando somente $3,4 \%$ de fêmeas com tal comportamento. Porto-Filho et al. (2005) registraram, em $10 \%$ dos estros, fêmeas que não demonstraram qualquer comportamento de aceitação de monta, sendo tais casos caracterizados como estros silenciosos.

A ovulação ocorre após o final do estro. Dobson \& Kamonpatana (1986) observaram que as búfalas ovulam, em média, cerca de 10 horas após o final do estro. De maneira geral, a maioria das búfalas ovula dentro de 24 a 48 horas após o início do estro, ou 6 a 21 horas após o final do estro (Siqueira et al., 2009).

\section{Avaliação da dinâmica folicular em bubalinos}

A foliculogênese é definida como o processo de formação, crescimento e maturação folicular, iniciando-se com a formação do folículo primordial e finalizando-se com o estádio de folículo maduro ou pré-ovulatório (Lucy et al., 1992; Picton, 2001; Siqueira et al., 2009). O estudo da dinâmica folicular, nos diferentes momentos do ciclo estral, pode ajudar a elucidar fenômenos envolvidos com procedimentos de sincronização do ciclo estral e ovulação, assim como na resposta ovariana de búfalas superovuladas, colaborando para o aumento da fertilidade (Baruselli et al., 1997; Oba \& Souza, 2003; Siqueira et al., 2009)

Os trabalhos mais consistentes sobre o estudo da dinâmica folicular foram realizados através dos exames ultrassonográficos dos ovários. O advento da ultrassonografia em tempo real possibilitou estudar com maior facilidade e detalhamento o crescimento folicular, sem a necessidade de se abater os animais experimentais. Com essa técnica foi possível avaliar o surgimento das estruturas ovarianas e correlacioná-las com os eventos endocrinológicos em tempo real no decorrer do ciclo estral (Ginther et al., 1989; Lucy et al., 1992; Baruselli et al., 1997).

$\mathrm{O}$ primeiro trabalho relacionado à dinâmica folicular durante o ciclo estral em búfalas foi realizado por Singh et al. (1984), que observaram a presença de estruturas ovarianas durante o ciclo estral em novilhas da raça Surti. Manik et al. (1994), através do acompanhamento da morfologia ovariana, relataram uma maior ocorrência $(83,3 \%)$ de animais com duas ondas de crescimento folicular e menor ocorrência $(16,7 \%)$ de animais com três ondas foliculares. Enquanto que, Taneja et al. (1996), observaram a ocorrência de duas e uma onda de crescimento folicular durante 7 ciclos estrais onde nenhuma búfala foi observada com três ondas. Esses resultados demonstraram que as búfalas apresentavam comportamento folicular semelhante ao apontado para bovinos (Siqueira et al., 2009).

Sabe-se que o número de ondas foliculares em cada ciclo é correlacionado com o comprimento do ciclo, que, por sua vez, depende da meia-vida do corpo lúteo. Desta forma, ciclos estrais de uma onda estão associados com uma curta fase luteal e caracterizados por curtos comprimentos do ciclo estral (Baruselli et al., 1997; Siqueira et al., 2009). No estudo de Baruselli et al. (1997), foi reportado que apenas uma búfala apresentou uma única onda de crescimento folicular, caracterizada pelo ciclo estral de curta duração (13 dias). No entanto, o ciclo subsequente desse animal apresentou duração normal (22 dias), sendo que o ciclo estral curto encontrado deveria estar relacionado a uma fase luteal de curta duração no período pós-parto. De fato, esses autores registraram maior incidência de búfalas com ciclos de duas ondas de crescimento folicular $(63,3 \%)$ e observaram que as fêmeas que apresentaram três ondas de crescimento folicular tiveram a fase luteal mais longa, maior intervalo interovulatório e maior duração do ciclo estral (Siqueira et al., 2009).

Em búfalas que apresentam duas ondas de crescimento folicular por ciclo estral, a primeira e a segunda ondas iniciaram-se nos dias 1,8 $\pm 0,6 \mathrm{e}$ $7,8 \pm 2,0$ do ciclo, respectivamente (Taneja et al., 
1996), e estes autores registraram valores de 15,5 $\pm 0,9 \mathrm{~mm}$ para o diâmetro máximo do folículo dominante, com taxa média de crescimento de 1,0 $\pm 0,1 \mathrm{~mm} /$ dia. Resultados semelhantes foram registrados no estudo de Baruselli et al. (1997), no qual o início da primeira e da segunda ondas de crescimento folicular ocorreu nos dias $1,16 \pm 0,5$ e $10,83 \pm 1,09$, respectivamente. Animais com três ondas de crescimento folicular apresentam o início da primeira, segunda e terceira ondas nos dias 1,10 $\pm 0,32,9,30 \pm 1,25$ e $16,80 \pm 1,22$, respectivamente. Manik et al. (1994) encontraram diâmetro máximo do folículo dominante de 13,8 \pm $0,37 \mathrm{~mm}$ e taxa de crescimento de $0,94 \pm$ $0,12 \mathrm{~mm} /$ dia durante $11,8 \pm 1,39$ dias.

Gimenes et al. (2007) avaliaram a divergência folicular por meio de ultrassonografia, sendo que o diâmetro dos dois maiores folículos foi de 7,2 \pm $0,3 \mathrm{~mm}$ e de $6,4 \pm 0,3 \mathrm{~mm}$ para o folículo dominante e o subordinado, respectivamente, no início do desvio folicular, e a capacidade ovulatória foi adquirida quando o folículo dominante alcançou 8,5 $\mathrm{mm}$. Desse modo, observa-se que importantes modificações no padrão de crescimento folicular podem ser observadas por meio da ultrassonografia em bubalinos, tal como é observado em bovinos (Siqueira et al., 2009).

\section{Emprego da inseminação artificial (IA) em bubalinos}

Entre as biotecnologias da reprodução aplicadas aos bubalinos, a inseminação artificial (IA) tem contribuído significativamente para o melhoramento genético. A IA é a primeira biotecnologia aplicada para se melhorar a reprodução e a genética, e permanece como o principal veículo para a disseminação rápida de genes valiosos, sendo o método de escolha para se melhorar a qualidade genética dos animais de produção (Vishwanath \& Shannon, 2000; Swelum et al., 2011).

No Brasil, as primeiras pesquisas envolvendo a inseminação artificial (IA) em bubalinos foram conduzidas em duas regiões geográficas bastante distintas, na região Sudeste e na região Norte (Oba, 1990; Vale et al., 1991; Vale \& Ohashi, 1994). Para se obter sucesso nesta biotécnica, como nos bovinos, torna-se de fundamental importância a detecção correta do estro (cio). Portanto, a prática desta observação é viável em pequenos lotes. Em grandes áreas com rebanhos maiores, torna o manejo extremamente trabalhoso e ineficiente. Dessa forma, alguns cuidados devem ser tomados para a obtenção de bons resultados na detecção do estro, tais como: responsabilidade, habilidade e conhecimento específico do observador; número de observações diárias (mínimo duas) e tempo dedicado a cada uma delas (60 minutos); número de rufiões/búfalas (máximo 1/25); utilização de rufiões de grande porte, dominância e bom libido; número de búfalas por lote de observação (60 animais); e manejar calmamente os animais sem causar estresse durante a observação (Baruselli, 1993).Além desses fatores relacionados ao manejo reprodutivo, deve-se atentar para outros, como cuidados sanitários, nutrição, matrizes e recursos humanos.

Para a implantação da IA, torna-se necessário um manejo sanitário efetivo, com vacinações e vermifugações e um bom manejo nutricional, pois a nutrição influencia no balanço energético, tendo como consequência, repetição de cio, aumento no período de serviço e perdas embrionárias. $\mathrm{O}$ melhor momento para a inseminação da búfala é após o término do estro, quando a mesma rejeita a monta do rufião, fato que dificulta o processo da IA, em função da necessidade de manter na propriedade pelo menos dois rufiões, um no pasto para detecção do estro e outro no curral para detecção do término do estro (Ohashi et al 2006).

A aceitação de monta varia de 6 a 24 horas e as observações de estro são realizadas a cada 12 horas, a búfala pode ser inseminada de 12 a 24 horas após a detecção do estro. As búfalas que manifestarem estro somente em um período de observação devem ser inseminadas obedecendo o protocolo utilizado em bovinos, ou seja, as búfalas que são observadas em estro pela manhã devem ser inseminadas na tarde do mesmo dia, e as búfalas observadas em estro à tarde devem ser inseminadas na manhã do dia seguinte, bem cedo. Por outro lado, as búfalas que apresentarem estros de maior duração, detectados em mais de um período de observação, devem ser inseminadas quando não aceitarem mais a monta (Vale \& Ohashi,1994).

Baruselli (1999) realizou, no período entre 1993 e 1995, 831 inseminações em dez propriedades localizadas na região do Vale do Ribeira, litoral sul do Estado de São Paulo. Segundo este autor, as taxas de concepção médias da primeira, segunda e terceira IA foram de $51,11 \%, 55,72 \%$ e $52,38 \%$, respectivamente. Neste mesmo estudo, observou uma grande 
variação de resultados em relação ao número de doses de sêmen/concepção $(1,64$ a 2,47$)$ e com relação à taxa de concepção $(60,68$ a 40,44\%) conforme a propriedade. As variações observadas podem ser explicadas pelas diferenças de manejo nos rebanhos.

\section{Utilização da inseminação artificial em tempo fixo (IATF) em bubalinos}

A utilização de protocolos de fácil execução e que não necessitem de identificação de estro podem contribuir para o incremento da utilização da inseminação artificial em bubalinos. Os protocolos consistem em sincronizar o crescimento folicular e a ovulação, permitindo o uso da IATF em todos os animais da propriedade, mesmo naqueles que não estejam manifestando estro ou ciclicidade, colaborando, assim, para o aumento do emprego desta biotecnologia em bubalinos e o melhoramento genético para carne e leite seja promovido mais rapidamente e com maior eficiência (Baruselli et al., 2009).

Em bubalinos, desde o final da década de noventa, têm sido realizadas pesquisas com o objetivo de avaliar a eficácia de diferentes protocolos de sincronização do estro e indução da ovulação para IATF. Os dados são indicativos de que é possível obter resultados satisfatórios com o emprego dessa biotécnica. No entanto, observa-se que a estacionalidade reprodutiva desta espécie pode interferir na eficiência dos protocolos de IATF (Baruselli, 1999; Baruselli et al., 1999).

Baruselli et al. (2009) observaram que, durante a estação reprodutiva favorável (outono e inverno), em rebanhos com alta taxa de ciclicidade, as búfalas respondem adequadamente ao protocolo Ovsynch 40 a 60 dias pós parto com boas taxas de concepção (48,8\%): No dia 0 administração por via intramuscular (i.m) de $20 \mu \mathrm{g}$ GnRH (5 ml de Acetato de Buserelina/Conceptal ${ }^{\circledR}$, Hoescht Roussel Veterinária S.A. São Paulo $\mathrm{SP}$ ); Dia 7 administração por via i.m de $150 \mu \mathrm{g}$ de Prostaglandina (d-cloprostenol/ Prosolvin ${ }^{\circledR}$, Intervet, Curitiba - PR) e no dia 9 administração por via i.m de $10 \mu \mathrm{g}$ de GnRH (2,5 ml Acetato de Buserelina/Conceptal ${ }^{\circledR}$, Hoescht Roussel Veterinária S.A. São Paulo - SP) , a IATF é realizada 16 a 24 horas após a última administração do GnRH (Figura 1) (Baruseli, 1999). Entretanto, fora da estação reprodutiva favorável (entre a primavera e o verão), a maioria das búfalas manifestam prolongados períodos de anestro e não respondem adequadamente ao tratamento com $\mathrm{GnRH}$, com baixas taxas de concepção $(6,9 \%, \mathrm{P}<0,05)$. Nesse período, estes autores verificaram que estes animais respondem somente a protocolos baseados em progesterona $\mathrm{e}$ estrógenos (Benzoato de Estradiol; BE). Dessa forma, torna-se importante diferenciar os protocolos para serem utilizados em búfalas de acordo com a estação reprodutiva e o estado de ciclicidade.

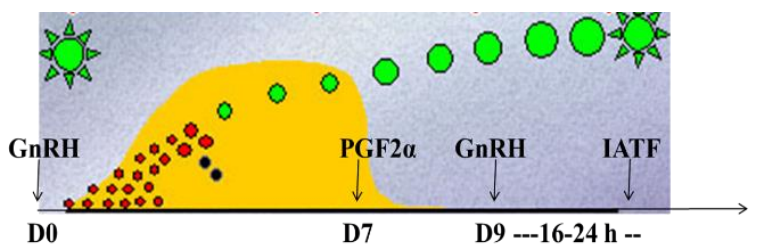

Figura 1. Protocolo Ovsynch para IATF em bubalinos durante a estação reprodutiva favorável.

Baruselli \& Carvalho (2005) em estudo com 967 búfalas submetidas ao protocolo de sincronização da ovulação (Ovsynch) durante a estação reprodutiva favorável (março a agosto), obtiveram taxa de concepção média de $48,8 \%$. Neste trabalho, os autores observaram a influência do escore de condição corporal na taxa de concepção $(\leq 3,0=31,4 \% ; 3,5=52,9 \% ; \mathrm{e} \geq 4,0=$ $57,1 \%$ ), sugerindo que as búfalas devem apresentar condição corporal entre 3,5 e 4,0 para obtenção de boa eficiência do tratamento a ser utilizado para IATF. O número de partos também interferiu na taxa de concepção, sendo que as primíparas apresentaram menor taxa do que as multíparas $(35,5 \%$ e $51,0 \%$, respectivamente), devendo-se preferencialmente sincronizar as multíparas para melhorar a eficiência dos tratamentos. O período pós-parto em que se iniciou o tratamento e a ordem da inseminação não interferiu na taxa de concepção, demonstrando que o tratamento pode ser iniciado entre 40 e 60 dias pós-parto, e os animais que não se tornarem gestantes à primeira sincronização podem ser tratados novamente. Desta forma, pode-se alcançar taxa de prenhez de aproximadamente $75 \%$ com duas inseminações sincronizadas em um período de serviço inferior a 100 dias (Baruselli \& Carvalho, 2005).

Estes dados corroboram com as informações de Zicarelli (1997) ao relatar que, mesmo com a estimulação hormonal exógena, os bubalinos continuam apresentando marcada estação reprodutiva. Diante dos dados apresentados, podese observar que a utilização da IATF com sincronização da ovulação em bubalinos pelo método Ovsynch é perfeitamente viável. 
A obtenção de melhores resultados ressalta-se que o método deve ser utilizado em animais com boa condição corporal, preferencialmente multíparas e durante a estação reprodutiva favorável, que seria de março a agosto (Baruselli et al., 1999; Baruselli et al., 2009).

Além do protocolo Ovsyncy, existem no mercado outros hormônios para tratamentos de sincronização do estro e da ovulação, tais como produtos eficientes que liberam progestágenos, que podem ser implantados na vagina $\left(\mathrm{CIDR}^{\circledR}\right.$, PRID $^{\circledR}$, PRIMER $^{\circledR}$ ) ou via auricular subcutânea $\left(\right.$ CRESTAR $^{\circledR}$ e SYNCROMATE B ${ }^{\circledR}$ ).

Baruselli (1999) relatou que os trabalhos realizados visando sincronizar o estro e a ovulação em bubalinos progestágenos têm apresentado baixa eficiência em búfalas leiteiras, com taxa de concepção de 11,4 a $29,2 \%$, com pequeno percentual de ovulações e com grande variabilidade no tempo de ovulação após a retirada do implante. O mesmo autor sugere que novos estudos devem ser realizados na tentativa de inserir a estes protocolos fatores que aumentem as taxas de ovulação e de sincronização, com o objetivo de incrementar a eficiência dos tratamentos e viabilizá-los para o emprego a campo em larga escala.

\section{Superovulação (SOV) e transferência de embriões (TE) em bubalinos}

A técnica da superovulação (SOV) foi introduzida na espécie bubalina por Drost et al. (1983), e desde então tem sido aplicada, mesmo que experimentalmente, na maioria dos países onde há rebanhos de búfalos (Ohashi et al., 2006). Vários pesquisadores têm feito estudos com o objetivo de se verificar as respostas aos tratamentos superovulatórios em bubalinos com o Hormônio Folículo Estimulante (FSH) e a Gonadotrofina Coriônica Equina (eCG). Alguns trabalhos relatam que a administração do eCG não é uma boa opção para uso em protocolos de superovulação de búfalas devido a sua longa meia vida biológica, aumentando o estrógeno circulante e alterando o movimento das tubas uterinas, consequentemente afetando a maturação embrionária. Entretanto, os resultados apresentados, quando comparados aos de bovinos, tem sido inconsistente (Karaivanov, 1986; Misra et al.; 1999, Drost et al., 1983; Ohashi et al., 2006; Drost, 2007; Carvalho et al., 2011; Pereira et al., 2016).
Em geral as búfalas respondem bem ao protocolo de superovulação, apresentando boa taxa de ovulação e formação de corpos lúteos, apesar de ocorrer uma grande variação individual na resposta superovulatória (Ohashi et al., 2006). Entretanto, o principal fator para o insucesso da superovulação em bubalinos está relacionado à baixa taxa de recuperação embrionária (Baruselli et al., 1999. A razão da baixa taxa de recuperação embrionária em bubalinos é desconhecida, bem como o destino dos embriões (Ohashi et al., 2006). Segundo Baruselli (1997), esses embriões podem ser perdidos na cavidade abdominal ou ficarem retidos nas dobras do endométrio dificultando o seu deslocamento pela lavagem uterina. Além disso, a falta de ovulação ou a alta incidência de cistos foliculares em búfalas submetidas à superovulação também podem ser a causa dessa baixa taxa de recuperação embrionária (Zicarelli, 1994).

De acordo com Misra (1993), diversos fatores têm contribuído para a baixa taxa de recuperação embrionária, tais como falta de seleção baseada na capacidade reprodutiva tanto para as doadoras como para receptoras, falta de conhecimento sobre a dosagem e metabolismo das gonadotrofinas e falta de um protocolo definido de superovulação e IA. Adicionalmente, outros fatores como a reprodução sazonal e a falta de informações sobre o melhor momento para realização da lavagem uterina também parecem influenciar o resultado da superovulação (Ohashi et al., 2006).

Segundo Boni (1994), outro fator que pode estar relacionado à baixa taxa de recuperação é o aumento do tamanho ovariano devido ao alto estímulo hormonal provocado pela superovulação, o que dificultaria a captação do oócito pela tuba uterina. Para testar esta hipótese, Baruselli et al. (2002) realizaram um experimento de superovulação utilizando baixa concentração hormonal com o objetivo de evitar o aumento ovariano exagerado e facilitar sua captura pela tuba uterina. Obtiveram, assim, 2,8 corpos lúteos por ovário, bem abaixo da média citada por Misra et al. (1999), que foi cerca de 5 a 6 corpos lúteos por ovário. Após lavagem uterina, os autores ainda obtiveram baixa taxa de recuperação, que variou de 23,5 a $31,6 \%$, mesmo sem haver um grande aumento do tamanho ovariano, concluindo que a hipótese da dificuldade na captura do oócito pela tuba uterina não foi o fator responsável pela baixa taxa de recuperação embrionária. 
Mais estudos são necessários para tentar se esclarecer as causas da baixa taxa de recuperação embrionária em bubalinos. Além desses fatores, torna-se necessário o controle do estro e da ovulação da doadora e a sincronização do estro das receptoras para uma boa eficiência da técnica (Baruselli et al., 1999). Em outras espécies, tais como bovinos, ovinos, caprinos, equinos e carnívoros, já se produzem embriões in vitro, mas em búfalas deve-se melhorar e aumentar a eficiência da inseminação artificial e transferência de embriões (Crozet et al., 1987; Farstad, 2000; Oba \& Camargos, 2011; Ohashi et al., 2017).

\section{Produção in vitro (PIV) de embriões bubalinos}

Apesar das técnicas de superovulação e transferência de embriões na espécie bubalina estarem sendo bastante investigadas através dos esforços de vários grupos de pesquisa (Gasparrini, 2002), resultados consistentes que assegurem seu uso comercial no rebanho ainda não foram alcançados. A PIV ainda tem apresentado algumas limitações, tais como a quantidade e qualidade dos oócitos coletados por ovário, que são muito inferiores aos das fêmeas bovinas (Ohashi et al., 2006).

O número de oócitos obtidos por ovário de animais abatidos em matadouro descrito na literatura varia de 0,73 a 2,2 (Ohashi et al., 2003). Com relação à qualidade dos oócitos, Ohashi et al. (2003) observaram, em material de matadouro, que o número de oócitos com cumulus compacto foi cerca de três vezes maior em bovinos do que em bubalinos. De fato, observa-se que a qualidade dos oócitos obtidos é inferior no bubalino quando comparado ao bovino, e o mesmo tem sido observado com oócitos aspirados pelo método de OPU (Gonçalves et al., 2008).

Um dos fatores decisivos para o sucesso da PIV é o número de folículos antrais disponíveis para a aspiração folicular, número este que está intimamente relacionado às características reprodutivas da espécie e que no caso dos bubalinos apresentam um baixo número de folículos antrais, semelhante à espécie taurina (Manjunatha et al., 2007; Sartori \& Barros, 2011; Baldrighi et al., 2014) e cerca de duas vezes menos que em animais zebuínos (Bos indicus) (Ohashi et al., 2003; Saliba et al., 2013; Ohashi et al., 2017). Entretanto, não apenas a quantidade de oócitos obtidos por aspiração folicular é menor, como também a qualidade dos oócitos viáveis para PIV é inferior. Essas características têm como consequência uma menor taxa de blastocisto e menor taxa de prenhez por aspiração folicular por doadora, resultando na elevação do custo da gestação na referida espécie (Ohashi et al. (2017).

Ohashi et al. (2003) avaliaram a média de folículos e oócitos em ovário bovino e bubalino, e observaram que, em bovinos, de 8,7 $\pm 5,9$ folículos aspirados, se recuperaram 4,1 $\pm 2,6$ oócitos, com 2,9 $\pm 2,3$ células do cumulus compactas. Já em bubalinos, estes números foram $4,5 \pm 1,9,2,2 \pm 1,1$ e $0,9 \pm 0,8$, respectivamente. Também Ohashi et al. (2017), ao relatar alguns dados comparativos de programas de produção in vitro de embrião bubalino e bovino (Nelore) em duas fazendas no Estado do Pará, observaram, em 142 vacas aspiradas, 1.746 oócitos recuperados e 162 embriões, resultando em 69 gestações. Em 103 búfalas aspiradas, estes números foram $533 \mathrm{e}$ 19 , respectivamente, não resultando em nenhuma gestação.

Sá Filho et al. (2009) administrou somatrotopina recombinante bovina (bST) em búfalas com o objetivo de aumentar o número de folículos recrutados e a qualidade oocitária, melhorando assim a eficiência da técnica de aspiração folicular em fêmeas bubalinas. Esses autores observaram que o bST aumentou o número de folículos $(12,2$ versus 8,$7 ; \mathrm{P}<0,05)$ e o número de oócitos recuperados (5,2 versus 4,1 ; $\mathrm{P}=0,07$ ), não afetando a qualidade destes, bem como a sua capacidade de desenvolvimento. Desse modo, eles concluíram que o tratamento com bST pode aumentar o número de folículos recrutados por onda de crescimento folicular, demonstrando seu potencial em aumentar a eficiência da técnica de aspiração folicular na espécie bubalina.

A pressão de aspiração também pode comprometer a qualidade dos oócitos de bubalinos, visto que a adesão das células do cumulus a zona pelúcida parece não ser tão forte como nos bovinos (Boni, 1994). Este fato pode levar, dependendo da pressão exercida pelo sistema de aspiração folicular, a dispersão dessas células, comprometendo assim a qualidade do processo de maturação oócitária, influenciando negativamente na fecundação e também no desenvolvimento embrionário in vitro (Nandi et al., 1998; Ohashi et al., 2006). Assim, a qualidade do complexo cumulus-oocito parece interferir no processo de maturação e na qualidade dos oócitos produzidos in vitro (Gonçalves et al., 2008). 
Apesar dos esforços dos pesquisadores do mundo todo, foram obtidos poucos bezerros bubalinos produzidos inteiramente pelo processo de PIV. A primeira descrição da técnica de fertilização in vitro (FIV) em bubalino foi realizada por Singh et al. (1989), que utilizaram oócitos maturados in vitro, fecundados com sêmen congelado e cultivados in vitro até o estágio de mórula. Pouco tempo depois, Suzuki et al. (1991) relataram a primeira gestação em búfalas com embrião produzido in vitro, e Madan et al. (1992) relataram o primeiro nascimento (Ohashi et al., 2006).

A primeira tentativa de utilização de PIV, que associa as técnicas de maturação oocitária (MIV), fecundação in vitro (FIV) cultivo embrionário in vitro (CIV) e aspiração folicular (OPU), foi realizada por Boni et al. (1994), com resultados promissores (Ohashi et al., 2006). Desde o primeiro nascimento de bubalino por FIV, foram publicados inúmeros artigos sobre sistemas de produção in vitro de embriões, demonstrando os efeitos de diferentes protocolos e meios sobre o desenvolvimento de oócitos e embriões (Mondadori et al., 2010; Oba \& Camargos, 2011).

No Brasil, Baruselli \& Carvalho (2005) relataram o nascimento de dois bezerros bubalinos produzidos pelo processo de PIV. Mais de 100 trabalhos foram publicados entre 1991 e 2008, apresentando altas taxas de maturação (80\%), moderadas taxas de clivagem (50\%) e taxas moderadas de formação de blastocistos (20\%) na espécie bubalina (Suresh et al., 2009). Os relatos de eficácia de tratamentos são, em alguns casos, contraditórios, onde alguns estudos afirmam a existência de efeitos, enquanto outros não (Singhal et al., 2009; Oba \& Camargos, 2011; Ferraz et al., 2015; Kumar et al., 2015; Bhardwaj et al., 2016; Fu et al., 2016; Pereira et al., 2016; Ohashi et al., 2017).

A produção in vitro de embriões bubalinos apresenta alta taxa de maturação $(80 \%)$, moderada taxa de clivagem $(50 \%)$ e taxa moderada de formação de blastocistos (20\%) (Oba \& Camargos, 2011). Embora bovinos e bubalinos sejam espécies bem diferentes, observam-se que as técnicas desenvolvidas são as mesmas, podendo-se aproveitar o trabalho já realizado em bovinos para benefício dos bubalinos. No entanto, algumas restrições biológicas e fisiológicas dos bubalinos devem ser observadas no que tange à aplicação da PIV, sendo esta, limitada pelo número e qualidade dos oócitos, pelas baixas taxas de prenhez e pela alta perda de gestação póstransferência dos embriões, tornando a técnica mais cara que nos bovinos (Oba \& Camargos, 2011).

Apesar do progresso nos processos de aspiração folicular, MIV e FIV, há a necessidade de aprimoramentos nas diferentes etapas do processo de PIV. Umas das medidas seria a elaboração de meios mais adequados e específicos para os processos de capacitação espermática e cultivo dos gametas e embriões. A utilização de animais em boas condições de manejo nutricional e sanitário também auxiliaria no aprimoramento da técnica, pois resultam em um blastocisto de melhor qualidade, melhorando desta forma a taxa de prenhez e o desenvolvimento normal da gestação. Deste modo, a técnica da PIV poderá se tornar realmente uma técnica eficiente para a produção de embriões na espécie bubalina (Oashi et al., 2006).

A criopreservação de células reprodutivas é uma tecnologia em desenvolvimento que tem muitas implicações para o avanço rápido das pesquisas em diversas espécies, como nos bubalinos. No entanto, estes apresentam uma baixa capacidade reprodutiva, evidenciada, entre outros fatores, por mudanças nas proteínas do acrossoma e danos na membrana durante o congelamento do sêmen. Neste sentido, diversos estudos têm sido relacionados aos protocolos de criopreservação de sêmen, com algumas tentativas feitas para que este processo seja mais eficiente nesses animais a fim de se melhorar a sua eficiência reprodutiva (Totey et al., 1992; Leibfried-Rutledge et al., 1997; Nugent et al., 1997; Wani et al., 2004).

Sabe-se que um número substancial de espermatozoides, cerca de 50\%, é danificado durante o processo de criopreservação. Isto é devido à cristalização intracelular que ocorre durante esse processo, o que danifica as organelas e membranas do espermatozoide, induzindo mudanças na morfologia, capacitação espermática e reação acrossomal, que podem causar morte celular (Mazur, 1984; Watson, 2000; Garner et al., 2001; Swelum et al., 2011). Devido a isso, os protocolos de criopreservação devem minimizar os efeitos deletérios aos espermatozoides (Kumar et al., 2003). Segundo Mudgal (1992), diluidores com glicerol-gema (como o TRIS ${ }^{\circledR}$ ) com uma concentração de 6,5 a 7,0\% de glicerol parecem ser os criodiluentes mais efetivos para o sêmen de búfalo, e o nitrogênio líquido foi observado como 
sendo o método mais efetivo para a criopreservação de sêmen em bubalinos.

O espermatozoide bubalino difere do espermatozoide bovino em muitos aspectos (Lambrechts et al., 1999). De acordo com Mudgal (1992), os espermatozoides do búfalo são mais sensíveis do que os do bovino aos danos de membrana, principalmente da membrana acrossomal, durante o processo de criopreservação. Desse modo, são necessários protocolos de criopreservação diferenciados e adaptados à espécie bubalina devido às suas particularidades (Lambrechts et al., 1999).

O processo de criopreservação causa danos aos espermatozoides, e devido a isso, torna-se necessário a adição de um crioprotetor. Neste sentido, sabe-se que a composição dos crioprotetores e diluidores disponíveis são fatores importantes para o sucesso da criopreservação (Curry et al., 1994; Dhami et al., 1996; Guthrie et al. 2002).

O uso de crioprotetores é essencial para a viabilidade do espermatozoide após o congelamento pelo fato de minimizarem a formação de cristais de gelo intracelular, a sua adição e subsequente remoção pode proporcionar um potencial dano osmótico à célula. Durante a adição de um crioprotetor à suspensão celular, as células são expostas a um ambiente hiperosmótico, sendo que mudanças na osmolaridade podem resultar em danos na integridade funcional da membrana plasmática ou mesmo em morte celular (Watson, 1995; Gao et al., 1997; Medeiros et al., 2002; Swelum et al., 2011).

Entre os crioprotetores utilizados para a proteção do sêmen, o glicerol tem sido usado extensivamente em várias espécies de animais domésticos. Entretanto, pode causar efeitos osmóticos e tóxicos na membrana plasmática e no metabolismo das células criopreservadas, sendo que altas concentrações podem levar à morte celular (Jeyendran et al., 1985; Hammerstedt \& Graham, 1992; Buhr et al., 2001). Devido a isso, o etileno glicol tem sido utilizado em substituição ao glicerol em muitas espécies, devido ao fato desse crioprotetor penetrar mais rapidamente na membrana plasmática do que o glicerol, resultando em um menor choque osmótico às células durante os processos de congelação e descongelação (Gilmore et al., 2000; Guthrie et al., 2002). Aparentemente, o etileno glicol tem menos efeitos nocivos sobre a viabilidade e motilidade do espermatozoide, fornecendo melhores efeitos de proteção ao acrossoma espermático do que o glicerol (Ball \& Vo et al., 2001; Swelum et al., 2011).

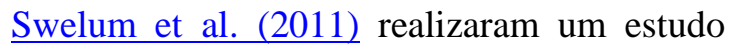
com o objetivo de avaliar os efeitos da substituição do glicerol pelo etileno glicol na criopreservação do sêmen bubalino, e observaram melhorias na viabilidade espermática, integridade do acrossoma e da membrana plasmática e nas taxas de prenhez quando usaram 5 ou $7 \%$ de etileno glicol em substituição a $10 \%$ de glicerol. Do mesmo modo, Abdel-Khalek et al. (2008) demonstraram que a congelação de sêmen bubalino com 5 ou $7 \%$ de etileno glicol foi melhor quando comparado com $10 \%$ de glicerol no diluidor, indicando que o etileno glicol pode proteger o sêmen bubalino significativamente melhor do que glicerol. Já o diluidor TRIS $^{\circledR}$-gema tem sido amplamente recomendado para a rotina de uso na criopreservação do sêmen bubalino, devido à manutenção da motilidade durante o processo de criopreservação e à viabilidade do sêmen pósdescongelação (Miyasaki et al., 2014). Contudo, a congelabilidade e as taxas de fertilidade do sêmen bubalino são menores do que o sêmen bovino, quando os mesmos são criopreservados com o diluidor TRIS ${ }^{\circledR}$-gema (Kumar et al., 1994). Desse modo, ainda são necessários mais estudos para se identificar o melhor diluidor para a criopreservação do sêmen capaz de produzir taxas de fertilidade aceitáveis em bubalinos (Sansone et al., 2001; Andrabi, 2009; Akhter et al., 2010)

Miyasaki et al., (2014) realizaram um estudo a fim de comparar a utilização de diluidores como o TRIS $^{\circledR}$-gema, Ringer-Lactato e Leite desnatado na criopreservação do sêmen bubalino, e observaram que os melhores valores para motilidade espermática pós-descongelação e vigor foram obtidos com a utilização do TRIS $^{\circledR}$-gema. Do mesmo modo, Rasul et al. (2000) obtiveram os melhores resultados para motilidade espermática pós-descongelação e integridade das membranas plasmática e acrossomal com a utilização do TRIS $^{\circledR}$-gema no processo de criopreservação do sêmen bubalino.

Dhami et al. (1996) também realizaram um trabalho a fim de avaliar a utilização de dois diluidores (TRIS ${ }^{\circledR}$-gema e Leite desnatado) na criopreservação do sêmen bubalino, e observaram que esses diluidores não diferiram com relação aos parâmetros de congelabilidade, longevidade pósdescongelação e taxas de fertilidade, indicando 
que diluidores à base de leite podem ser usados com eficiência em substituição ao TRIS ${ }^{\circledR}$-gema nos processos de criopreservação de sêmen bubalino (Dhami et al., 1993). No entanto, no estudo de Dhami et al. (1996), a motilidade do sêmen pós-descongelação foi menor com a utilização do leite desnatado em comparação ao TRIS ${ }^{\circledR}$-gema, o que pode ter sido devido à rápida deterioração da capacidade do poder tampão e ao aumento do crescimento microbiano observado com o leite desnatado, o que contribui para a elevação da acidez do meio biológico e redução da sobrevivência espermática.

Diluidores comercialmente disponíveis baseados em lecitina de soja, tais como AndroMed $^{\circledR}$, Biociphos plus ${ }^{\circledR}$ e Bioxcell ${ }^{\circledR}$, têm sido usados para a criopreservação do sêmen de bovinos, caprinos e ovinos (Aires et al., 2003; Amirat et al., 2005; Muino et al., 2007). Alguns autores observaram que a criopreservação do sêmen bovino com o uso de Bioxcell ${ }^{\circledR}$ foi capaz de manter a qualidade seminal (motilidade e integridade das membranas plasmática e acrossomal) e produzir taxas de fertilidade aceitáveis (Gil et al., 2003; Hansen et al., 2005; Celeghini et al., 2008). Neste sentido, Akhter et al. (2010) realizaram um estudo a fim de comparar a utilização do diluidor comercial Bioxcell ${ }^{\circledR}$ com o TRIS $^{\circledR}$-gema na qualidade pós-descongelação e na fertilidade in vivo do sêmen bubalino. Esses autores observaram que a motilidade espermática, viabilidade, integridade das membranas plasmática e acrossomal e anomalias espermáticas não diferiram entre os diluidores Bioxcell ${ }^{\circledR} \mathrm{e}$ TRIS $^{\circledR}$-gema. Também foi observado no estudo de Akhter et al. (2010) que as taxas de fertilidade in vivo do sêmen bubalino criopreservado em Bioxcell $^{\circledR}$ e TRIS $^{\circledR}$-gema foram similares, indicando que os diluidores comercialmente disponíveis, como o Bioxcell ${ }^{\circledR}$, podem ser usados com sucesso para a criopreservação do sêmen bubalino.

Alguns autores têm relatado que, em bovinos, o Bioxcell ${ }^{\circledR}$ tem a habilidade para manter o conteúdo de glutationa no sêmen criopreservado porque sua formulação contém maiores concentrações desse antioxidante do que o TRIS ${ }^{\circledR}$ Gema. Como tem sido relatada uma relação positiva entre os níveis de glutationa e a qualidade seminal, é sugerida que a melhoria na qualidade pós-descongelação do sêmen bovino criopreservado em Bioxcell ${ }^{\circledR}$ possa ser atribuída à maior concentração de glutationa, que protege o esperma contra o estresse oxidativo e ação das espécies reativas ao oxigênio (Gil et al., 2000; Gadea et al., 2004; Stradaioli et al., 2007). No entanto, para o sêmen bubalino, o Bioxcell ${ }^{\circledR}$ não tem sido capaz de melhorar a qualidade seminal quando comparado ao TRIS $^{\circledR}$-gema. No trabalho de Akhter et al. (2010) foi sugerido que o espermatozoide bubalino é mais propenso ao estresse oxidativo devido ao maior conteúdo de fosfolipídios poli-insaturados, sendo que, desse modo, o nível de glutationa no Bioxcell ${ }^{\circledR}$ usado nesse estudo não foi suficiente para proteger o esperma do estresse oxidativo durante os processos de congelação e descongelação (Sansone et al., 2001). Outros estudos com diferentes concentrações e formas de uso dos antioxidantes devem ser executados para se obter mais informações sobre outros parâmetros espermáticos e sobre a fertilidade do sêmen bubalino. Além disso, mais estudos são necessários acerca da criopreservação de sêmen em bubalinos e suas implicações na fertilidade desses animais.

\section{Conclusões}

A eficiência de várias biotécnicas da reprodução em bubalinos, tanto em machos quanto em fêmeas, ainda é baixa e de difícil execução, devido às diferenças biológicas, produtivas e do ambiente técnico, e muitas dificuldades provêm de origem genética, devido à baixa pressão por características como fertilidade e sazonalidade reprodutiva. Ainda não está claro se as baixas taxas de prenhez após a transferência de embriões são devido à qualidade dos embriões ou às receptoras, que falham em estabelecer ou manter a gestação.

Apesar de várias biotécnicas estarem sendo aplicadas em machos e fêmeas, mais estudos relacionados à fisiologia reprodutiva, como a produção de sêmen e a dinâmica folicular, são necessários para se aperfeiçoarem os protocolos de criopreservação de sêmen e de sincronização do estro e ovulação para IATF, pois muitos destes ainda estão sendo utilizados do mesmo modo como são em bovino.

Além disso, observa-se que somente a IA tem apresentado resultados consistentes, ou seja, relação custo benefício favorável, o que assegura sua aplicação a nível comercial, já as demais biotécnicas, apesar de apresentarem resultados promissores, ainda necessitam de aprimoramentos que as tornem viáveis para uso em rotina 
comercial, com o objetivo de se aumentar a eficiência reprodutiva do rebanho bubalino.

\section{Referências}

Abdel-khalek, E. A., Shalaby, N. A., Eid, L. N. \& Gbr, A. A. 2008. Effect of various types and levels of cryoprotectants on sperm motility during freezing processes of Egyptian buffalo semen. Journal of Agricultural Science, 33, $7159-7171$.

Aires, V. A., Hinsch, K. D., Mueller-Schloesser, F., Bogner, K., Mueller-Schloesser, S. \& Hinsch, E. 2003. In vitro and in vivo comparison of egg yolk-based and soybean lecithin-based extenders for cryopreservation of bovine semen. Theriogenology, 60, 269279.

Amirat, L., Anton, M., Tainturier, D., Chatagnon, G., Battut, I. \& Courtens, J. L. 2005. Modifications of bull spermatozoa induced by three extenders: Biociphos, low density lipoprotein and Triladyl, before, during and after freezing and thawing. Reproduction, 129, 535-543.

Andrabi, S. M. H. 2009. Factors affecting the quality of cryopreserved buffalo (bubalus bubalis) bull spermatozoa. Reproduction in Domestic Animals, 44, 552-569.

Akhter, S., Ansari, M. S., Rakha, B. A., Andrabi, S. M. H., Iqbal, S. \& Ullah, N. 2010. Cryopreservation of buffalo (Bubalus bubalis) semen in Bioxcell® extender. Theriogenology, 74, 951-955.

Baldrighi, J. M., Sá Filho, M. F., Batista, E. O. S., Lopes, R. N. V. R., Visintin, J. A., Baruselli, P. S. \& Assumpcão, M. E. O. A. 2014. AntiMullerian hormone concentration and antral ovarian follicle population in Murrah heifers compared to Holstein and Gyr kept under the same management. Reproduction in Domestic Animals, 49, 1015-1020.

Ball, B. A. \& Vo, A. 2001. Osmotic tolerance of equine spermatozoa and the effects of soluble cryoprotectants on equine sperm motility, viability, and mitochondrial membrane potential. Journal of Andrology, 22, 10611069.

Bhardwaj, R., Ansari, M. M., Parmar, M. S., Chandra, V. \& Sharma, G. T. 2016. Stem cell conditioned media contains important growth factors and improves in vitro buffalo embryo production. Animal Biotechnology, 27, 118125.
Baruselli, P. S. 1993. Manejo reprodutivo de bubalinos. São Paulo: SAA/Coordenadoria da Pesquisa Agropecuária: Instituto de Zootecnia/EEZ-Vale do Ribeira, 46p.

Baruselli, P. S., Mucciolo, R. G., Visintin, J. A., Viana, W. G., Arruda, R. P., Madureira, E. H., Oliveira, C. A. \& Molero-Filho, J. R. 1997. Ovarian follicular dynamics during the estrous cycle in buffalo (Bubalus bubalis). Theriogenology, 47, 1531-1547.

Baruselli, P. S. 1999. Inseminação artificial em tempo fixo com sincronização da ovulação em bubalinos. In: Bubalinos: Sanidade, reprodução e produção. Ed. Barnabé, V.H. Jaboticabal: FUNEP, 202p.

Baruselli, P. S., Madureira, E. H., Visintin, J. A.; Barnabe, V. H.; Barnabe, R. C. \& Amaral, R. 1999. Inseminação artificial em tempo fixo com sincronização da ovulação em bubalinos. Revista Brasileira de Reprodução Animal, 23, 360-362.

Baruselli, P. S., Marques, M. O., Arruda, R. P., Carvalho, N. A. T. \& Oliveira, C. A. 2002. Plasma estradiol and progesterone concentrations in superovulated buffalo in presence of CIDRB devices. Theriogenology, 57, 761-770.

Baruselli, P. S. \& Carvalho, N. A. T. 2005. Biotecnologias da reprodução em bubalinos (Bubalus bubalis). Revista Brasileira de Reprodução Animal, 29, 417-425.

Baruselli, P. S., Gimenes, L. U. \& Sales, J. N. S. Fisiologia reprodutiva de fêmeas taurinas e zebuínas. Revista Brasileira de Reprodução Animal, 31, 205-211.

Baruselli, P. S., Carvalho, N. A. T. \& Jacomini, J. O. 2009. Eficiência do uso da inseminação artificial em búfalos. Revista Brasileira de Reprodução Animal, 6, 104-110.

Boni, R. 1994. In vitro embryo production in bovine and buffalo species. Buffalo Journal, Suplemento 2, 147-157.

BRASIL. Ministério da Agricultura, Pecuária e Abastecimento (MAPA). Rebanho bubalino brasileiro em 2017. Disponível em www.agricultura.gov.br. Acesso em 05 de Maio de 2018.

Buhr, M. M., Fiser, P., Bailey, J. L. \& Curtis, E. F. 2001. Cryopreservation in different concentrations of glycerol alters boar sperm and their membranes. Journal of Andrology, 22, 961-969. 
Carvalho, N. A. T., Bombonato, P. P., D'Angelo, M. \& Baruselli, P. S. 2011. Caracterização anatomofuncional do Sistema genital de fêmeas bubalinas (Bubalus bubalis) e suas implicações na múltipla ovulação e transferência de embriões. Revista Brasileira de Reprodução Animal, 35, 95-103, 2011.

Celeghini, E. C. C., Arruda, R. P., Andrade, A. F. C.; Nascimento, J., Rapahel, C. F. \& Rodrigues, P. H. M. 2008. Effects that bovine sperm cryopreservation using two different extenders has on sperm membranes and chromatin. Animal Reproduction Science, 104, 119-131.

Crozet, N., Huneau, D., Desmedt, V., Théron, M. C., Szollosi, D., Torrès, S. \& Sévellec, C. 1987. In vitro fertilization with normal development in the sheep. Gamete Research, 16, 159-170.

Curry, M. R., Millar, J. D. \& Watson, P. F. 1994. Calculated optimal cooling rates for ram and human sperm cryopreservation fail to conform with empirical observations. Biology of Reproduction, 51, 1014-1021.

Dhami, A. J., Mohan, G. \& Sahni, K. L. 1993. Effect of extenders and additives on preservability of cattle and buffalo semen at $5^{\circ} \mathrm{C}$ and $196{ }^{\circ} \mathrm{C}$. Indian Journal of Animal Science, 63, 492-498.

Dhami, A. J., Sahni, K. L, Mohan, G. \& Vani, V. R. 1996. Effects of different variables on the freezability, post-thaw longevity and fertility of buffalo spermatozoa in the tropics. Theriogenology, 46, 109-120.

Dobson, H. \& Kamonpatana, M. 1986. A review of female cattle reproduction with special reference to a comparison between buffaloes, cows and zebu. Journal of Reproduction and Fertility, 77, 1-36.

Drost, M., Wright Jr., J. M., Cripe, W. S. \& Richter, A. R. 1983. Embryo transfer in water buffalo (Bubalus bubalis). Theriogenology, 20, 579-584.

Drost, M. 2007. Advanced reproductive technology in the water buffalo. Theriogenology, 68, 450-453.

Farstad, W. 2000. Current state in biotechnology in canine and feline reproduction. Animal Reproduction Science, 60-61, 375-387.

Ferraz, M. L., Sá Filho, M. F., Batista, E. O. S., Watanabe, Y. F., Watanabe, M. R., Dayan, A., Joaquim, D .C., Accorsi, M. R., Gimenes, L. U., Vieira, L. M. \& Baruselli, P. S. 2015.
Paradoxical effects of bovine somatotropin treatment on the ovarian follicular population and in vitro embryo production of lactating buffalo donors submitted to ovum pickup. Animal Reproduction Science, 154, 1-7.

Fu, Q., Liu, Z. F., Huang, Y. L., Lu, Y. Q. \& Zhang, M. 2016. Comparative proteomic analysis of mature and immature oocytes of Swamp Buffalo (Bubalus bubalis). International Journal of Molecular Sciences, 17, 1-10.

Gadea J., Sellés, E., Marco, M. A., Coy, P., Matás, M., Romar, R. \& Ruiz, S. 2004. Decrease in glutathione content in boar sperm cryopreservation: Effect of the addition of reduced glutathione to the freezing and thawing extenders. Theriogenology, 62, 690701.

Gao, D. Y., Mazur, P. \& Critser, J. K. 1997. Fundamental cryobiology of mammalian spermatozoa. In: Karow, A.M.; Critser, J.K (eds.). Reproductive Tissue Banking: Scientific Principles. San Diego: Academic Press, 263328.

Garner, D. L., Thomas, G. A., Gravance, C. G., Marshall, C. E., DeJarnette, J. M. \& Allen, C. H. 2001. Seminal plasma addition attenuates the dilution effect in bovine sperm. Theriogenology, 56, 31-40.

Gasparrini, B. 2002. In vitro embryo production in buffalo specie: state of the art. Theriogenology, 57, 237-256.

Gil, J., Januskauskas, A., Haard, M. C. H., Haard, M. G. M., Johanisson, A., Söderquist, L. \& Rodriguez-Mártinez, H. 2000. Functional sperm parameters and fertility of bull semen extended in Biociphos Plus. Reproduction in Domestic Animals, 35, 69 -77.

Gil, J., Rodriguez-Irazoqui, M., Lundeheim, N. \& Rodriguez-Mártinez, H. 2003. Fertility of ram semen in Bioexcell and used for cervical artificial insemination. Theriogenology, 59, 1157-1170.

Gilmore, J. A., Liu, J., Woods, E. J., Peter, A. T. \& Critser, J. K. 2000. Cryoprotective agent and temperature effects on human sperm membrane permeabilities: convergence of theoretical and empirical approaches for optimal cryopreservation methods. Human Reproduction, 15, 335-343.

Gimenes, L. U., Carvalho, N. A. T., Sá Filho, M. F., Torres-Júnior, J. R. S., Ayres, H., Vannucci, F. S., Bianconi, L. L., Bisinotto, R. S., 
Reichert, R. H., Beltran, M. P., Nogueira, G. P. $\&$ Baruselli, P. S. 2007. Follicle selection by ultrasonography and plasmatic characteristics and ovulatory capacity in buffaloes. Italian Journal of Animal Science, 6, 629-631.

Ginther, O. J., Kastelic, J. P. \& Knopf, L. 1989. Composition and characteristics of follicular waves during the bovine estrus cycle. Animal Reproduction Science, 20, 187-200.

Gonçalves, P. B. D., Figueiredo, J. R. \& Freitas, V. J. F. 2008. Biotécnicas Aplicadas a Reprodução Animal. 2. ed. São Paulo: Roca, $395 \mathrm{p}$.

Guthrie, H. D., Liu, J. \& Critser, J. K. 2002. Osmotic tolerance limits and effects of cryoprotectants on motility of bovine spermatozoa. Biology of Reproduction, 67, 1811-1816.

Hammerstedt, R. H. \& Graham, J. K. 1992. Cryopreservation of poultry sperm: the enigma of glycerol. Cryobiology, 29, 26-38.

Hansen, G. B. B., Morris, I. D., Ersboll, A. K., Greve, T. \& Christensen, P. 2005. DNA integrity in sexed bull sperm assessed by neutral Comet assay and sperm chromatin structure assay. Theriogenology, 63, 17891802.

Instituto Brasileiro de Geografia e Estatística IBGE. Diretoria de Pesquisas, Coordenação e Agropecuária. Pesquisa da Pecuária Municipal em 2016. Disponível em www.ibge.gov.br. Acesso em 05 de julho de 2018.

Jainundeen, M. R. 1986. Reproduction in water buffalo. In: Morrow, D.A. Current therapy in theriogenology: diagnosis, treatment and prevention of reproduction diseases in animals. Philadelphia, USA, W.B. Saunders, 443-449.

Jeyendran, R. S., Van Der Ven, H. H., PerezPalaez, M. \& Zaneveld, L. J. D. 1985. Nonbeneficial effects of glycerol on the oocyte penetrating capacity of cryopreserved and incubated human spermatozoa. Cryobiology, 22, 434-437.

Karaivanov, C. H. 1986. Comparative studies on the superovulatory effect of PMSD and FSH in water buffalo. Theriogenology, 26 51-59.

Kumar, S., Sahni, K. L. \& Mohan, G. 1994. Effect of yolk, glycerol and sugars on post-thaw survival of buffalo spermatozoa in TRIS dilutor. Indian Journal Animal Science, 64, 362-364.
Kumar, S., Millar, J. D. \& Watson, P. F. 2003. The effect of cooling rate on the survival of cryopreserved bull, ram, and boar spermatozoa: a comparison of two controlledrate cooling machines. Cryobiology, 46, 24-53.

Kumar, P., Verma, A., Kumar, M., De, S., Kumar., R. \& Datta, T. K. 2015. Expression pattern of glucose metabolism genes correlate with development rate of buffalo oocytes and embryos in vitro under low oxygen condition. Journal of Assisted Reproduction and Genetics, 32, 471-478.

Lambrechts, H. 1999. The effect of cryopreservation on the survivability, viability and motility of epididymal African buffalo (Syncerus caffer) spermatozoa. Theriogenology, 52, 1241-1249.

Leibfried-Rutledge, M. L, Dominko, T., Cristser, E. S. \& Critser, J. K. 1997. Tissue maturation in vivo and in vitro: gamete and early embryo ontogeny. In: Karow, A.M.; Critser, J.K., editors. Reproductive Tissue Banking, Scientific Principles. San Diego Academic Press, 23-111.

Lucy, M. C., Savio, J. D., Badinga, L, De La Sota, R. L. \& Thatcher, W. W. 1992. Factors that affect ovarian follicular dynamics in cattle. Journal of Animal Science, 70, 3615-26,

Malhado, C. H. M., Malhado, A. C. M., Carneiro, P. L. S., Ramos, A. A., Carrillo, J. A. \& Pala, A. 2013. Inbreeding depression on production and reproduction traits of buffaloes from Brazil. Animal Science Journal, 84, 289-295.

Madan et al. (1992)

Manik, R. S., Madan, M. L. \& Singla, S. K. 1994. Ovarian follicular dynamics in water buffaloes (Bubalus bubalis): ultrasonically monitoring individual follicles for wave hypothesis. Theriogenology, 41, 246.

Manjunatha, B. M., Gupta, P. S. P., Ravindra, J. P., Devaraj, M., Ramesh, H. S. \& Nandi, S. 2007. In vitro developmental competence of buffalo oocytes collected at various stages of the estrous cycle. Theriogenology, 68, 882888.

Mazur, P. 1984. Freezing of living cells: mechanisms and implications. American Journal of Physiology, 247, 125-142.

Medeiros, C. M., Forell, F., Oliveira, A. T. D. \& Rodrigues, J. L. 2002. Current status of sperm cryopreservation: why isn't it better? Theriogenology, 57, 327-344. 
Mello, R. R. C., Ferreira, J. E., Silva, A. P. T. B. \& Mello, M. R. B. 2013. Desenvolvimento folicular inicial em bovinos. Revista Brasileira de Reprodução Animal, 37, 328-333.

Misra, A. K. 1993. Superovulation and embryo transfer in buffalo; process, problems and future prospects in India. Buffalo Journal, 1, 13-24.

Misra, A. K., Mutha Rao, M., Kasiraj, R., Ranga, N. S., Reddy, N. S. \& Pant, H. C. 1999. Factors affecting pregnancy rate following nonsurgical embryo transfer in buffalo (Bubalus bubalis): A retrospective study. Theriogenology, 52, 110.

Miyasaki, M. Y. A., Vale, W. G., Baía, F. A. C., Minervino, A. H. H., Ribeiro, H. F. L. \& Miyasaki, M. T. A. 2014. Comparação entre o Tris, Lactose/Tris, Ringer-Lactato e Leite Desnatado como diluidores na criopreservação do sêmen bubalino. Revista Brasileira de Medicina Veterinária, 36, 1-10.

Mondadori, R. G., Santin, T. R., Fidélis, A. A. G., Name, K. P. O., Silva, J. S., Rumpf, R. \& Bao, S. N. 2010. Ultrastructure of in vitro oocyte maturation in buffalo (Bubalus bubalis). Zygote, 18, 309-314.

Mudgal, V. 1992. Reproduction in River Buffaloes. In: Tulloh, N.M.; Holmes, J.H.G (Ed's). Buffalo Production. New York: Elsevier, 171-181.

Muino, R., Fernández, M. \& Peña, A. 2007. Postthaw survival and longevity of bull spermatozoa frozen with an egg yolk-based or two egg yolk-free extenders after an equilibration period of $18 \mathrm{~h}$. Reproduction in Domestic Animals, 42, 305-311.

Nandi, S., Chauan, M. S. \& Palta, P. 1998. Influence of cumulus cells and sperm concentration on cleavage rate and subsequent embryonic development of buffalo (Bubalus bubalis) oocytes matured and fertilized in vitro. Theriogenology, 50, 1251-1262.

Nugent, D., Meirow, D., Brook, P. F., Aubard, Y. \& Gosden, R. G. 1997. Transplantation in reproductive medicine: previous experience, present knowledge and future prospects. Human Reproduction Update, 3, 267-280.

Oba, E. 1990. UNESP Domina Técnica de Inseminar Búfalas. Jornal O Estado de São Paulo, Suplemento Agrícola, 1808.

Oba, E. 1993. Tópicos atualizados ligados à reprodução na espécie bubalina. In: Sanidade e produtividade em búfalos. ed. Molero Filho, J. et al. Jaboticabal, FUNEP, 202p.

Oba, E. \& Souza, M. I. L. 2003. Avanços na reprodução de bovinos e bubalinos. In: Contribuição ao estudo dos bubalinos: período de 1972-2001: UNESP, Botucatu-SP. Palestras, Botucatu, SP: FMVZ/UNESP, 541564.

Oba, E. \& Camargos, A. S. 2011. Produção in vitro de embriões bubalinos. Revista Brasileira de Reprodução Animal, 35, 80-87.

Ohashi, O. M., Miranda, M. S., Sousa, J. S., Sousa, A. J. O., Cordeiro, M. S. \& Biondi, F. C. 2003. Produção in vitro de embriões bubalinos. Revista Brasileira Reprodução Animal, 27, 103-108.

Ohashi, O. M., Cordeiro, M. S. \& Miranda, M. S. 2006. Biotecnologia da reprodução aplicada aos bubalinos. Revista de Ciências Agrárias, $45,1-14$.

Ohashi, O. M., Almeida, N. N. C., Cordeiro, M. S., Rolim Filho, S. T., Ribeiro, H. F. L., Santos, A. X., Ayala, H. M. D., Brito, V. C., Ramos, A. S., Silva, T. V. G., Santos, S. S. D. \& Miranda, M. S. 2017. Produção in vitro de embrião (PIVE) na espécie bubalina. Revista Brasileira Reprodução Animal, 41, 195-200.

Pereira, E. C. M., Borges, A. M. \& Oba, E. 2016. Maturação in vitro de oócitos bubalinos e seu efeito sobre o desenvolvimento embrionário. Revista Brasileira Reprodução Animal, 40, 4350.

Picton, H. M. 2001. Activation of follicle development: the primordial follicle. Theriogenology, 55, 1193-1210.

Porto-Filho, R. M., Baruselli, P. S. \& Madureira, E. H. 2005. Uso da radiotelemetria para detecção do estro em fêmeas búfalas: luteólise durante duas fases do ciclo estral, ultrassonografia da ovulação e perfis hormonais. Boletim de Sociedade Brasileira de Medicina Veterinária, 1, 13-32.

Rasul, Z., Anzar, M., Jalali, S. \& Ahmad, N. 2000. Effect of buffering systems on post thaw motion characteristics, plasma membrane integrity, and acrosome morphology of buffalo spermatozoa. Animal Reproduction Science, 59, 31-41.

Rodrigues, C. F. C., Lapichini, J. E. C. B., Liserre, A. M., Souza, K. B., Fachini, C. \& Reichert, R. H. 2008. Oportunidades e desafios da bubalinocultura familiar da região Sudoeste 
Paulista. Revista Tecnologia e Inovação Agropecuária, 100-109.

Rohilla, R. K., Tuli, R. K. \& Goyal, R. L. 2005. Comparative study of the effects of cryoprotective agents in freezing Murrah buffalo bull semen. Indian Journal of Veterinary Research, 14, 37-43.

Sá Filho, M. F., Carvalho, N. A. T., Gimenes, L. U., Torres-Júnior, J. R., Nasser, L. F. T., Tonhati, H., Garcia, J. M., Gasparrini, B., Zicarelli, L. \& Baruselli, P. S. 2009. Effect of recombinant bovine somatotropin (bST) on follicular population and on in vitro buffalo embryo production. Animal Reproduction Science, 113, 51-59.

Saliba, W., Gimenes, L. U., Drumond, R., Bayão, H., Alvim, M., Baruselli, P., Bastianetto, E., Leite, R. \& Gasparrini, B. 2013. Efficiency of OPU-IVEP-ET of fresh and vitrified embryos in buffaloes. Buffalo Bulletin, 32, 385-388.

Sansone, G., Nastri, M. J. F. \& Fabbrocini, A. 2001. Storage of buffalo (Bubalus bubalis) semen. Animal Reproduction Science, 62, 5576.

Santana Júnior M. L., Aspilcueta-Borquis, R. R., Bignardi, A. B., Albuquerque, L. G. \& Tonhati, H. 2011. Population structure and effects of inbreeding on Milk yield and quality of Murrah bufalloes. Journal of Dairy Science, 94, 52045211.

Sartori, R. \& Barros, C. M. Reproductive cycles in Bos indicus cattle. 2011. Animal Reproduction Science, 124, 244-250.

Singh, G., Totey, S. M. \& Talwar, G. P. 1989. In vitro fertilization of buffalo (Bubalus bubalis) oocytes matured in vitro. Theriogenology, 31, 255, Abstract.

Singh, G., Singh, B. C., Sharma, S. S. \& Sharma, R. D. 1984. Studies on oestrus symptoms of buffalo heifers (Murrah buffalo). Theriogenology, 21, 849-858.

Singhal, S., Prasad, S., Singh, B., Prasad, J. K. \& Gupta, H. P. 2009. Effect of including growth factors and antioxidants in maturation medium used for in vitro culture of buffalo oocytes recovered in vivo. Animal Reproduction Science, 113, p.4450.

Siqueira, J. B., Leal, L. S. \& Oba, E. 2009. Dinâmica folicular ovariana na espécie bubalina. Revista Brasileira de Reprodução Animal, 33, 139-148.
Stradaioli, G., Noro, T., Sylla, L. \& Monaci, M. 2007. Decrease in glutathione (GSH) content in bovine sperm after cryopreservation: comparison between two extenders. Theriogenology, 67, 1249-1255.

Suresh, K. P., Nandi, S. \& Mondal, S. 2009. Factors affecting laboratory production of buffalo embryos: A meta-analysis. Theriogenology, 72, 978-985.

Suzuki, T., Singla, S. K., Sujata, J. \& Madan, M. L. 1991. Cleavage capability of water buffalo follicular oocytes classified by cumulus cells and fertilized in vitro. Journal of Veterinary Medical Science, 53, 475-478, 1991.

Swelum, A. A., Mansour, H. A., Esayed, A. A. \& Amer, H. A. 2011. Comparing ethylene glycol with glycerol for cryopreservation of buffalo bull semen in egg-yolk containing extenders. Theriogenology, 76, 833-842.

Taneja, M., Ali, A. \& Singh, G. 1996. Ovarian Follicular dynamics in water buffalo. Theriogenology, 46, 121-130.

Totey, S. M., Singh, G., Taneja, M., Pawshe, C. H. \& Talwar, G. P. 1992. In vitro maturation, fertilization and development of follicular oocytes from buffalo (Bubalus bubalis). Journal of Reproduction and Fertility, 95, 597607.

Tonhati, H., Muñoz, M. F. C., Oliveira, J. A., Duarte, J. M. C., Furtado, T. P. \& Tseimazides, S. P. 2000. Parâmetros Genéticos para a Produção de Leite, Gordura e Proteína em bubalinos. Revista Brasileira de Zootecnia, 29, 2051-2056.

Vale, W. G. 1988. Fisiologia da Reprodução na búfala (Bubalus bubalis). In: Vale, W. G. Bubalinos Fisiologia e Patologia da Reprodução. Campinas, Fundação Cargill, 138.

Vale, W. G., Ohashi, O. M., Ribeiro, H. F. L. \& Sousa, J. S. 1991. Semen freezing and artificial inseminations in amazon valley. Buffalo Journal, 7, 137-144.

Vale, W. G. \& Ohashi, O. M. 1994. Guia prático para inseminar bovinos e bubalinos. Belém: UFPA-CCB-CEBRAN, 24p.

Vale, W. G. \& Ribeiro, H. F. L. 2005. Características reprodutivas dos bubalinos: puberdade, ciclo estral, involução uterina e atividade ovariana no pós-parto. Revista Brasileira de Reprodução Animal, 29, 63-73. 
Vishwanath, R. \& Shannon, P. 2000. Storage of bovine semen in liquid and frozen state. Animal Reproduction Science, 62, 23-45.

Wani, N. A., Maurya, S. N., Misra, A. K., Saxena, V. B. \& Lakhchaura, B. D. 2004. Effect of cryoprotectants and their concentration on in vitro development of vitrified-warmed immature oocytes in buffalo (Bubalus bubalis). Theriogenology, 61, 831-842.

Watson, P. F. 2000. The causes of reduced fertility with cryopreserved semen. Animal Reproduction Science, 60-61, 481-492.

Zicarelli, L. 1994. Embryo Transfer in Mediterranean Buffaloes. Buffalo Journal, Suplemento 1, 87-101.
Zicarelli, L. \& Vale, W. G. 2002. Patrones reprodutivos estacionales y no estacionales en el búfalo doméstico. In: Berdugo, J. A.; Vale, W. G. (Ed.). Memorias del Curso Internacional de Reproducción Bufalina, Medellín, Colombia. Medellín: CATI, 33-58.

Recebido: 27 Julho, 2018

Aprovado: 27 Agosto, 2018

Publicado: 18 Outubro 2018

Licenciamento: Este artigo é publicado na modalidade Acesso Aberto sob a licença Creative Commons Atribuição 4.0 (CC-BY 4.0), a qual permite uso irrestrito, distribuição, reprodução em qualquer meio, desde que o autor e a fonte sejam devidamente creditados. 